\title{
Attribute-Based Encryption Optimized for Cloud Computing ${ }^{\star}$
}

\author{
Máté Horváth \\ mhorvath@crysys.hu \\ Laboratory of Cryptography and System Security (CrySyS Lab) $)^{\star \star}$
}

\begin{abstract}
In this work, we aim to make attribute-based encryption (ABE) more suitable for access control to data stored in the cloud. For this purpose, we concentrate on giving to the encryptor full control over the access rights, providing feasible key management even in case of multiple independent authorities, and enabling viable user revocation, which is essential in practice. Our main result is an extension of the decentralized CP-ABE scheme of Lewko and Waters [LW11] with identity-based user revocation. Our revocation system is made feasible by removing the computational burden of a revocation event from the cloud service provider, at the expense of some permanent, yet acceptable overhead of the encryption and decryption algorithms run by the users. Thus, the computation overhead is distributed over a potentially large number of users, instead of putting it on a single party (e.g., a proxy server), which would easily lead to a performance bottleneck. The formal security proof of our scheme is given in the generic bilinear group and random oracle models.
\end{abstract}

Keywords: storage in clouds, access control, attribute-based encryption, user revocation, multi-authority

\section{Introduction}

Recent trends show a shift from using companies' own data centres to outsourcing data storage to cloud service providers. Besides cost savings, flexibility is the main driving force for outsourcing data storage, although in the other hand it raises the issue of security, which leads us to the necessity of encryption. Traditional cryptosystems were designed to confidentially encode data to a target recipient (e.g. from Alice to Bob) and this seems to restrict the range of opportunities and flexibility offered by the cloud environment. Imagine the following scenario: some companies are cooperating on a cryptography project and from each, employees are working together on some tasks. Suppose that Alice wants to share some data of a subtask with those who are working on it, and with the managers of the project from the different companies. We see that encrypting this data with traditional techniques, causes that recipients must be determined formerly, moreover either they has to share the same private key or several encrypted versions (with different keys) must be stored. These undermine the possible security, efficiency and the flexibility which the cloud should provide.

Attribute-based encryption (ABE) proposed by Sahai and Waters [SW05] is intended for oneto-many encryption in which ciphertexts are encrypted for those who are able to fulfil certain requirements. The most suitable variant for fine-grained access control in the cloud is called ciphertext-policy (CP-)ABE, in which ciphertexts are associated with access policies, determined by the encryptor and attributes describe the user, accordingly attributes are embedded in the users' secret keys. A ciphertext can be decrypted by someone if and only if, his attributes satisfy the access structure given in the ciphertext, thus data sharing is possible without prior knowledge of who will be the receiver preserving the flexibility of the cloud even after encryption.

Returning to the previous example, using CP-ABE Alice can encrypt with an access policy expressed by the following Boolean formula: "CRYPtoProjeCt" AND ("SubTask Y" OR "MANAGER"). Uploading the ciphertext to the cloud, it can be easily accessed by the employees of each

* This is the full length version (last updated: January 5, 2015) of the conference paper, accepted for publication in the proceedings of SOFSEM 2015 (Editors: G.F. Italiano et al.), LNCS 8939, pp. 566-577, (c) Springer-Verlag Berlin Heidelberg 2015.

** Budapest University of Technology and Economics, Department of Networked Systems and Services, Magyar tudósok krt. 2, 1117 Budapest, Hungary. www.crysys.hu 
company, but the data can be recovered only by those who own a set of attributes in their secret keys which satisfies the access policy (e.g. "CRYPToProjeCT", "SubTAsk Y").

In spite of the promising properties, the adoption of CP-ABE requires further refinement. A crucial property of ABE systems is that they resist collusion attacks. In most cases (e.g. [BSW07Wat11] ) it is achieved by binding together the attribute secret keys of a specific user with a random number so that only those attributes can be used for decryption which contains the same random value as the others. As a result private keys must be issued by one central authority (CA) that would need to be in a position to verify all the attributes or credentials it issued for each user in the system. However even our example shows that attributes or credentials issued across different trust domains are essential and these have to be verified inside the different organisations (e.g. "MANAGER" attribute ). To overcome this problem, we are going to make use of the results of Lewko and Waters [WW1] about decentralising CP-ABE.

The other relevant issue is user revocation. In everyday use, a tool for changing a user's rights is essential as unexpected events may occur and affect these. An occasion when someone has to be revoked can be dismissal or the revealing of malicious activity. Revocation is especially hard problem in ABE, since different users may hold the same functional secret keys related with the same attribute set (aside from randomization). We emphasise that user revocation is applied in $e x-$ ceptional cases like the above-mentioned, as all other cases can be handled simpler, with the proper use of attributes (e.g. an attribute can include its planned validity like "CRYPTOPROJECT2015").

Related Work. The concept of ABE was first proposed by Sahai and Waters [SW05] as a generalization of identity-based encryption. Bethencourt et al. [BSW07] worked out the first ciphertext-policy ABE scheme in which the encryptor must decide who should or should not have access to the data that she encrypts (ciphertexts are associated with policies, and users' keys are associated with sets of descriptive attributes). This concept was further improved by Waters in Wat11.

The problem of building ABE systems with multiple authorities was first considered by Chase Cha07] with a solution that introduced the concept of using a global identifier (GID) for tying users' keys together. Her system relied on a central authority and was limited to expressing a strict AND policy over a pre-determined set of authorities. Decentralized ABE of Lewko and Waters [LW11 does not require any central authority and any party can become an authority while there is no requirement for any global coordination (different authorities need not even be aware of each other) other than the creation of an initial set of common reference parameters. With this it avoids placing absolute trust in a single designated entity, which must remain active and uncorrupted throughout the lifetime of the system. Several other multi-authority schemes (e.g. RNS11WLWG11]) were shaped to the needs of cloud computing, although these lack for efficient user revocation.

Attribute revocation with the help of expiring attributes was proposed by Bethencourt et al. BSW07. For single authority schemes Sahai et al. SSW12] introduced methods for secure delegation of tasks to third parties and user revocation through piecewise key generation. Ruj et al. [RNS11, Wang et al. WLWG11 and Yang et al. [YJRZ13] show traditional attribute revocation (in multi-authority setting) causing serious computational overhead, because of the need for key re-generation and ciphertext re-encryption. A different approach is identity-based revocation, two types of which were applied to the scheme of Waters Wat11. Liang et al. [LLLS10] gives the right of controlling the revoked set to a "system manager" while Li et al. [LZW ${ }^{+} 13$, follow [LSW10], from the field of broadcast encryption systems and give the revocation right directly to the encryptor. This later was further developed by Li et al. LXZ13 achieving full security with the help of dual system encryption. For this approach, but in key-policy ABE, Qian and Dong QD11 showed fully secure solution.

To the best of our knowledge no multi-authority system is integrated with identity-based user revocation and our work is the first in this direction.

Contribution. Based on [LW11] and [LSW10] we propose a scheme that adds identity-based user revocation feature to distributed CP-ABE. With this extension, we achieve a scheme with multiple, independent attribute authorities, in which revocation of specific users (e.g. with $I D_{i}$ ) from the system with all of their attributes is possible without updates of attribute public and secret keys (neither periodically, nor after revocation event). We avoid re-encryption of all ciphertexts the 
access structures of which contain a subset of attributes of the revoked user. The revocation right can be given directly to the encryptor, just like the right to define the access structure which fits to the cloud computing scenario.

Organization. In Section 2 we introduce the theoretical background that we use later and define the security of multi-authority CP-ABE schemes with ID-based revocation. In Section 3 the details of our scheme can be found together with efficiency and security analysis. Directions for further research are proposed in the last section.

\section{Background}

We first briefly introduce bilinear maps, give formal definitions for access structures and relevant background on Linear Secret Sharing Schemes (LSSS). Then we give the algorithms and security definitions of Ciphertext Policy Attribute-Based Encryption with identity-based user revocation.

\subsection{Bilinear maps}

We present the most important facts related to groups with efficiently computable bilinear maps.

Let $\mathbb{G}_{0}$ and $\mathbb{G}_{1}$ be two multiplicative cyclic groups of prime order $p$. Let $g$ be a generator of $\mathbb{G}_{0}$ and $e$ be a bilinear map (pairing), $e: \mathbb{G}_{0} \times \mathbb{G}_{0} \rightarrow \mathbb{G}_{1}$, with the following properties:

1. Bilinearity: $\forall u, v \in \mathbb{G}_{1}$ and $a, b \in \mathbb{Z}_{p}$, we have $e\left(u^{a}, v^{b}\right)=e(u, v)^{a b}$

2. Non-degeneracy: $e(g, g) \neq 1$.

We say that $\mathbb{G}_{0}$ is a bilinear group if the group operation in $\mathbb{G}_{0}$ and the bilinear map $e: \mathbb{G}_{0} \times \mathbb{G}_{0} \rightarrow$ $\mathbb{G}_{1}$ are both efficiently computable. Notice that the map $e$ is symmetric since $e\left(g^{a}, g^{b}\right)=e(g, g)^{a b}=$ $e\left(g^{b}, g^{a}\right)$.

\subsection{Access Structures}

Definition 1 (Access Structure [Bei96]).

Let $\left\{P_{1}, \ldots, P_{n}\right\}$ be a set of parties. A collection $\mathbb{A} \subseteq 2^{\left\{P_{1}, \ldots, P_{n}\right\}}$ is monotone if $\forall B, C$ : if $B \in \mathbb{A}$ and $B \subseteq C$ then $C \in \mathbb{A}$. An access structure (respectively, monotone access structure) is a collection (respectively, monotone collection) $\mathbb{A}$ of non-empty subsets of $\left\{P_{1}, \ldots, P_{n}\right\}$, i.e., $\mathbb{A} \subseteq 2^{\left\{P_{1}, \ldots, P_{n}\right\}} \backslash\{\emptyset\}$. The sets in $\mathbb{A}$ are called the authorized sets, and the sets not in $\mathbb{A}$ are called the unauthorized sets.

In our case the access structure $\mathbb{A}$ will contain the authorized sets of attributes, furthermore we restrict our attention to monotone access structures. However, it is possible to (inefficiently) realize general access structures using our techniques by having the not of attributes as separate attributes as well.

\subsection{Linear Secret Sharing Schemes (LSSS)}

To express the access control policy we will make use of LSSS. Here we adopt the definitions from those given in Bei96.

Definition 2 (Linear Secret Sharing Scheme). A secret-sharing scheme $\Pi$ over a set of parties $\mathcal{P}$ is called linear (over $\mathbb{Z}_{p}$ ) if

1. the shares for each party form a vector over $\mathbb{Z}_{p}$,

2. there exists a matrix $A$ with $\ell$ rows and $n$ columns called the share-generating matrix for $\Pi$. For all $i=1, \ldots, \ell$, the $i^{\text {th }}$ row of $A$ let the function $\rho$ defined the party, labelling row $i$ as $\rho(i)$. When we consider the column vector $v=\left(s ; r_{2}, \ldots, r_{n}\right)$, where $s \in \mathbb{Z}_{p}$ is the secret to be shared, and $r_{2}, \ldots, r_{n} \in \mathbb{Z}_{p}$ are randomly chosen, then $A v=\lambda$ is the vector of $\ell$ shares of the secret $s$ according to $\Pi$. The share $(A v)_{i}=\lambda_{i}$ belongs to party $\rho(i)$. 
In Bei96 it is shown that every linear secret sharing-scheme according to the above definition also enjoys the linear reconstruction property, defined as follows. Suppose that $\Pi$ is an LSSS for the access structure $\mathbb{A}$. Let $S \in \mathbb{A}$ be any authorized set, and let $I \subset\{1,2, \ldots, \ell\}$ be defined as $I=\{i \mid \rho(i) \in S\}$. Then, there exist constants $\left\{\omega_{i} \in \mathbb{Z}_{p}\right\}_{i \in I}$ such that, if $\left\{\lambda_{i}\right\}$ are valid shares of any secret $s$ according to $\Pi$, then $\sum_{i \in I} \omega_{i} \lambda_{i}=s$. Furthermore, it is also shown in Bei96] that these constants $\left\{\omega_{i}\right\}$ can be found in time polynomial in the size of the share-generating matrix $A$ and for unauthorized sets, no such $\left\{\omega_{i}\right\}$ constants exist.

We use the convention that $(1,0,0, \ldots, 0)$ is the "target" vector for any linear secret sharing scheme. For any satisfying set of rows $I$ in $A$, we will have that the target vector is in the span of $I$, but for any unauthorized set, it is not.

Using standard techniques (see [LW11] - Appendix G) one can convert any monotonic boolean formula into an LSSS representation. An access tree of $\ell$ nodes will result in an LSSS matrix of $\ell$ rows.

\subsection{Revocation Scheme for Multi-Authority CP-ABE}

A multi-authority Ciphertext-Policy Attribute-Based Encryption system with identity-based user revocation is comprised of the following algorithms:

Global $\operatorname{Setup}(\lambda) \rightarrow G P$ The global setup algorithm takes in the security parameter $\lambda$ and outputs global parameters $G P$ for the system.

Central Authority $\operatorname{Setup}(G P) \rightarrow\left(S K^{*}, P K^{*}\right)$ The central authority (CA) runs this algorithm with $G P$ as input to produce its own secret key and public key pair, $S K^{*}, P K^{*}$.

Identity $\operatorname{Key} \operatorname{Gen}\left(G P, R L, G I D, S K^{*}\right) \rightarrow K_{G I D}^{*}$ The central authority runs this algorithm upon a user request for identity secret key. It checks whether the request is valid and if yes (i.e. $G I D \notin R L)$, generates $K_{G I D}^{*}$ using the global parameters and the secret key of the CA.

Authority $\operatorname{Setup}(G P) \rightarrow(P K, S K)$ Each attribute authority runs the authority setup algorithm with $G P$ as input to produce its own secret key and public key pair, $S K, P K$.

$\operatorname{KeyGen}(G P, S K, G I D, i) \rightarrow K_{i, G I D}$ The attribute key generation algorithm takes in an identity $G I D$, the global parameters, an attribute $i$ belonging to some authority, and the secret key $S K$ for this authority. It produces a key $K_{i, G I D}$ for this attribute, identity pair.

$\operatorname{Encrypt}\left(G P, \mathcal{M},(A, \rho),\{P K\}, P K^{*}, R L\right) \rightarrow C T$

The encryption algorithm takes in a message $\mathcal{M}$, an access matrix $(A, \rho)$, the set of public keys for relevant authorities, the public key of the central authority, the revoked user list and the global parameters. It outputs a ciphertext $C T$.

$\operatorname{Decrypt}\left(G P, C T,(A, \rho),\left\{K_{i, G I D}\right\}, K_{G I D}^{*}, R L\right) \rightarrow \mathcal{M}$ The decryption algorithm takes in the global parameters, the revoked user list, the ciphertext, identity key and a collection of keys corresponding to attribute, identity pairs all with the same fixed identity GID. It outputs either the message $\mathcal{M}$ when the collection of attributes $i$ satisfies the access matrix corresponding to the ciphertext. Otherwise, decryption fails.

\subsection{Security Model}

We now define (chosen plaintext) security of multi-authority CP-ABE system with identity-based revocation. Security is defined using the following Security Game between an attacker algorithm $\mathcal{A}$ and a challenger. We assume that adversaries can corrupt authorities only statically, but key queries are made adaptively. The definition reflects the scenario where all users in the revoked set $R L$ get together and collude (this is because the adversary can get all of the private keys for the revoked set). The game is the following:

Setup. The challenger runs the Global Setup algorithm to obtain the global public parameters $G P$. $\mathcal{A}$ specifies a set $A A^{\prime} \subseteq A A$ of corrupt attribute authorities and uses the Authority Setup to obtain public and private keys. For honest authorities in $A A \backslash A A^{\prime}$ and for the Central Authority, the challenger obtains the corresponding keys by running the Authority Setup and Central Authority Setup algorithms, and gives the public keys to the attacker. 
Key Query Phase. $\mathcal{A}$ adaptively issues private key queries for identities $G I D_{k}$ (which denotes the $k^{\text {th }} G I D$ query). The challenger gives $\mathcal{A}$ the corresponding identity keys $K_{G I D}^{*}$ by running the Identity KeyGen algorithm. Let $U L$ denote the set of all queried $G I D_{k}$. $\mathcal{A}$ also makes attribute key queries by submitting pairs of $\left(i, G I D_{k}\right)$ to the challenger, where $i$ is an attribute belonging to a good authority. The challenger responds by giving the attacker the corresponding key, $K_{i, G I D_{k}}$.

Challenge. The attacker gives the challenger two messages $M_{0}, M_{1}$, a set $R L \subseteq U L$ of revoked identities and an access matrix $(A, \rho)$.

$R L$ and $A$ must satisfy the following constraints. Let $V$ denote the subset of rows of $A$ labelled by attributes controlled by corrupt authorities. For each identity $G I D_{k} \in U L$, let $V_{G I D_{k}}$ denote the subset of rows of $A$ labelled by attributes $i$ for which the attacker has queried $\left(i, G I D_{k}\right)$. For each $G I D_{k} \in U L \backslash R L$, we require that the subspace spanned by $V \cup V_{G I D_{k}}$ must not include $(1,0, \ldots, 0)$ while for $G I D_{k} \in R L$, it is allowed and we only require that the subspace spanned by $V$ must not include $(1,0, \ldots, 0)$. (In other words, the attacker cannot ask for a set of keys that allow decryption, in combination with any keys that can be obtained from corrupt authorities in case of a non revoked $G I D_{k}$. For revoked identities we only do not allow corrupted attributes to satisfy the access structure alone.)

The attacker must also give the challenger the public keys for any corrupt authorities whose attributes appear in the labelling $\rho$.

The challenger flips a random coin $\beta \in(0,1)$ and sends the attacker an encryption of $M_{\beta}$ under access matrix $(A, \rho)$ with the revoked set $R L$.

Key Query Phase 2. The attacker may submit additional attribute key queries $\left(i, G I D_{k}\right)$, as long as they do not violate the constraint on the challenge revocation list $R L$ and matrix $(A, \rho)$.

Guess. $\mathcal{A}$ must submit a guess $\beta^{\prime}$ for $\beta$. The attacker wins if $\beta^{\prime}=\beta$. The attacker's advantage in this game is defined to be $\mathbb{P}\left(\beta^{\prime}=\beta\right)-\frac{1}{2}$.

Definition 3. We say that a multi-authority CP-ABE system with identity-based revocation is (chosen-plaintext) secure (against static corruption of attribute authorities) if, for all revocations sets $R L$ of size polynomial in the security parameter, all polynomial time adversary have at most a negligible advantage in the above defined security game.

\section{Our Results}

To build our model we will use the prime order group construction of Lewko and Waters [LW11, because of its favourable property of having independent attribute authorities. In order to achieve identity-based revocation we supplement the distributed system with a Central Authority. However it seems to contradict with the original aim of distributing the key generation right, this additional authority would generate only secret keys for global identifiers $\left(G I D \in \mathbb{Z}_{p}\right)$ of users and the attribute key generation remains distributed. Our Central Authority does not possess any information that alone would give advantage during decryption, in contrast to single authority schemes, where the authority is able to decrypt all ciphertexts. Regarding this, we can say that our system remains distributed, in spite of launching a Central Authority.

Approach to the Cloud Storage Scenario. We give a high-level description about a possible application of the algorithms that we proposed in Subsection 2.4 (for graphichal depiction see Figure 11). Because of efficiency reasons data should be encrypted by a symmetric cipher, always using fresh random number as key, which is also encrypted, but with our scheme and in this form attached to the ciphertext that is stored by the cloud service provider (CSP). Decryption is possible for users, who can obtain the symmetric key, or with other words those, who possess the necessary attributes and were not revoked. Attribute Authorities are run locally on trusted servers of organisations, that are using the system, while the Central Authority is run by the CSP, which also maintains (archives, publishes) the $R L$ revocation list, based on the revocation requests from authorised parties from the organisations. The ABE encryption always uses the fresh $R L$ and $\mathrm{ABE}$ decryption is run with the $R L$ at the encryption time of the ciphertext, which are obtained from the CSP. This approach automatically leads to lazy re-encryption of ciphertext, as fresh symmetric key and $R L$ are used whenever data is edited. 


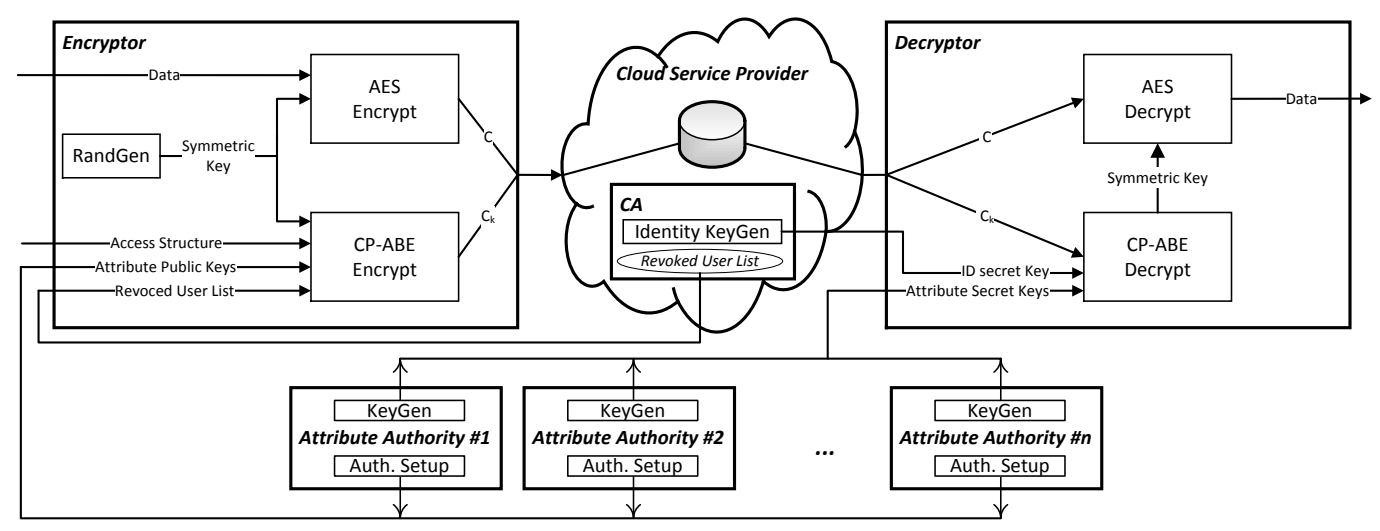

Fig. 1. A possible usage of the proposed ABE scheme for access control in a cloud storage scenario.

Our Technique. We face with the challenges of identity-based revocation. To realize the targeted features, we use some ideas from public key broadcast encryption systems [LSW10. A recent ${ }^{1}$ work of Cao and Liu [CL14] points out an inherent drawback of the [LSW10] scheme, namely that for malicious users it is worth to exchange their decryption keys in order to maximize their interests. However we utilize similar techniques as [LSW10], our system is not vulnerable to this kind of misuse, because unlike in broadcast encryption, where having a non-revoked secret key is the only requirement for decryption, in $\mathrm{ABE}$, users are also required to fulfil requirements related to their attributes. Thus such collusion could have only a restricted benefit ${ }^{2}$ as the set of ciphertexts that can be decrypted is also restricted by the used attribute secret keys (which cannot be mixed between different users) 3

We use secret sharing in the exponent. Suppose an encryption algorithm needs to create an encryption with a revocation set $R L=G I D_{1}^{*}, \ldots, G I D_{r}^{*}$ of $r$ identities. The algorithm will create an exponent $s^{*} \in \mathbb{Z}_{p}$ and split it into $r$ random shares $s_{1}, \ldots, s_{r}$ such that $\sum_{k=1}^{r} s_{k}=s^{*}$. It will then create a ciphertext such that any revoked user with $G I D_{k}^{*}$ will not be able to incorporate the $k^{\text {th }}$ share and thus not decrypt the message.

This approach presents the following challenges. First, we need to make crucial that the decryptor need to do the GID comparisons even if his attributes satisfy the access structure of the ciphertext. Second we need to make sure that a user with revoked identity $G I D_{k}^{*}$ cannot do anything useful with share $k$. Third, we need to worry about collusion attacks between multiple revoked users.

To address the first one we are going to take advantage of the technique of [LW11] that is used to prevent collusion attacks. Here the secret $s$, used for the encryption, is divided into shares, which are further blinded with shares of zero. This structure allows for the decryption algorithm to both reconstruct the main secret and to "unblind" it in parallel. If a user with a particular identifier $G I D$ satisfies the access structure, he can reconstruct $s$ in the exponent by raising the group elements to the proper exponents. This operation will simultaneously reconstruct the share of 0 and thus the $e(H(G I D), g)$ blinding terms will cancel out. When we would like to make this algorithm necessary, but not enough for decryption it is straightforward to spoil the "unblinding" of the secret by changing the shares of zero in the exponent to shares of an other random number, $s^{*} \in \mathbb{Z}_{p}$. Thus we can require an other computation, namely the comparison of the decryptor's

${ }^{1}$ CL14 appeared on ePrint some months later than our work.

2 Of course, when users reveal their secret keys, we cannot hope for security in any encryption method, but assuming honest users, it is their interest to keep the secrets. As long as the attributes of (still nonrevoked) colluding users do not cover all the access policies, our scheme will not reveal all ciphertexts for the malicious group.

${ }^{3}$ We also note that the flaw of [LSW10]'s security proof, mentioned by CL14] does not affect our results, as we use different proof technique. 
and the revoked users' GIDs. If correspondence is found, the algorithm stops, otherwise reveals the blinding, enabling decryption.

The second challenge is addressed by the following method. A user with $G I D \neq G I D_{k}^{*}$ can obtain two linearly independent equations (in the exponent) involving the share $s_{k}$, which he will use to solve for the share $s_{k}$. However, if $G I D=G I D_{k}^{*}$, the obtained equations are going to be linearly dependent and the user will not be able to solve the system.

In the third case, the attack we need to worry about is where a user with $G I D_{k}^{*}$ processes ciphertext share $l$, while another user with $G I D_{l}^{*}$ processes share $k$, and then they combine their results. To prevent collusion, we use $H(G I D)$ as the base of the identity secret key, such that in decryption each user recovers shares $s_{k} \cdot \log _{g} H(G I D)$ in the exponent, disallowing the combination of shares from different users.

\subsection{Our Construction}

To make the following notions more understandable, in Table 1 we summarize the new (compared to [LW11]) keys and variables which we introduce in our construction. Based on the above principles,

Table 1. The summary of our new notations

\begin{tabular}{ccl}
\hline Notation & Meaning & \multicolumn{1}{c}{ Role } \\
\hline$P K^{*}$ & $\left\{g^{a}, g^{1 / b}\right\}$ & public key of the Central Authority \\
$S K^{*}$ & $\{a, b\}$ & secret key of the Central Authority \\
$K_{G I D}^{*}$ & $H(G I D)^{(G I D+a) b}$ & global identity secret key of a user \\
$C_{1, k}^{*}$ & $\left(g^{a} g^{G I D_{k}^{*}}\right)^{-s_{k}}$ & revoked user identification in the ciphertext \\
$C_{2, k}^{*}$ & $g^{s_{k} / b}$ & secret share in the ciphertext \\
$R L$ & $\left\{G I D_{1}^{*}, \ldots, G I D_{r}^{*}\right\}$ & list of $r$ users, who should be revoked \\
\hline
\end{tabular}

the proposed algorithms are the following:

Global $\operatorname{Setup}(\lambda) \rightarrow G P$

In the global setup, a bilinear group $\mathbb{G}_{0}$ of prime order $p$ is chosen. The global public parameters, $G P$, are $p$ and a generator $g$ of $\mathbb{G}_{0}$, and a function $H$ mapping global identities $G I D \in \mathbb{Z}_{p}$ to elements of $\mathbb{G}_{0}$ (this is modelled as a random oracle in the security proof).

Central Authority $\operatorname{Setup}(G P) \rightarrow\left(S K^{*}, P K^{*}\right)$

The algorithm chooses random exponents $a, b \in \mathbb{Z}_{p}$, keeps them as secret key $S K^{*}=\{a, b\}$ and publishes $P K^{*}=\left\{g^{a}, g^{1 / b}\right\}$.

Identity KeyGen $\left(G P, R L, G I D, S K^{*}\right) \rightarrow K_{G I D}^{*}$

Upon the request of a user it first checks whether the user is on the list of revoked users $(R L)$ or it has been queried before, if yes refuses the request, otherwise computes $H(G I D)$ and generates the global identity secret key:

$$
K_{G I D}^{*}=H(G I D)^{(G I D+a) b} .
$$

Authority $\operatorname{Setup}(G P) \rightarrow(P K, S K)$

For each attribute $i$ belonging to the authority (these indices $i$ are not reused between authorities), the authority chooses two random exponents $\alpha_{i}, y_{i} \in \mathbb{Z}_{p}$ and publishes $P K=$ $\left\{e(g, g)^{\alpha_{i}}, g^{y_{i}} \forall i\right\}$ as its public key. It keeps $S K=\left\{\alpha_{i}, y_{i} \forall i\right\}$ as its secret key.

$\operatorname{KeyGen}(G P, S K, G I D, i) \rightarrow K_{i, G I D}$

To create a key for a $G I D$, for attribute $i$ belonging to an authority, the authority computes:

$$
K_{i, G I D}=g^{\alpha_{i}} H(G I D)^{y_{i}}
$$

$\operatorname{Encrypt}\left(G P, \mathcal{M},(A, \rho),\{P K\}, P K^{*}, R L\right) \rightarrow C T$

The encryption algorithm takes in a message $\mathcal{M}$, an $n \times \ell$ access matrix $A$ with $\rho$ mapping its rows to attributes, the global parameters, the public keys of the relevant authorities, the user identity public key and the most recent list of revoked users. 
It chooses random $s, s^{*} \in \mathbb{Z}_{p}$ and a random vector $v \in \mathbb{Z}_{p}^{\ell}$ with $s$ as its first entry. Let $\lambda_{x}$ denote $A_{x} \cdot v$, where $A_{x}$ is row $x$ of $A$. It also chooses a random vector $w \in \mathbb{Z}_{p}^{\ell}$ with $s^{*}$ as its first entry. Let $\omega_{x}$ denote $A_{x} \cdot w$.

For each row $A_{x}$ of $A$, it chooses a random $r_{x} \in \mathbb{Z}_{p}$ and supposed that the number of revoked users is $|R L|=r$ it chooses $s_{k}$ such that $s^{*}=\sum_{k=1}^{r} s_{k}$. The $C T$ ciphertext is computed as

$$
\begin{array}{cc}
C_{0}=\mathcal{M} \cdot e(g, g)^{s} \\
\forall x=1, \ldots, n: & C_{1, x}=e(g, g)^{\lambda_{x}} e(g, g)^{\alpha_{\rho(x)} r_{x}}, C_{2, x}=g^{r_{x}}, C_{3, x}=g^{y_{\rho(x)} r_{x}} g^{\omega_{x}}, \\
\forall k=1, \ldots, r: & C_{1, k}^{*}=\left(g^{a} g^{G I D_{k}^{*}}\right)^{-s_{k}}, C_{2, k}^{*}=g^{s_{k} / b}
\end{array}
$$

$\operatorname{Decrypt}\left(G P, C T,(A, \rho),\left\{K_{i, G I D}\right\}, K_{G I D}^{*}, R L\right) \rightarrow \mathcal{M}$

We assume the ciphertext is encrypted under an access matrix $(A, \rho)$. If the decryptor is not on the list of revoked users $(R L)$ and has the secret keys $K_{G I D}^{*}$ for his GID and $\left\{K_{i, G I D}\right\}$ for a subset of rows $A_{x}$ of $A$, such that $(1,0, \ldots, 0)$ is in the span of these rows, then the decryptor proceeds as follows. First chooses constants $c_{x} \in \mathbb{Z}_{p}$ such that $\sum_{x} c_{x} A_{x}=(1,0, \ldots, 0)$ and denoting $r=|R L|$ computes:

$$
\frac{\prod_{x}\left(\frac{C_{1, x} \cdot e\left(H(G I D), C_{3, x}\right)}{e\left(K_{\rho(x), G I D}, C_{2, x}\right)}\right)^{c_{x}}}{\prod_{k=1}^{r}\left(e\left(K_{G I D}^{*}, C_{2, k}^{*}\right) e\left(C_{1, k}^{*}, H(G I D)\right)\right)^{1 /\left(G I D-G I D_{k}^{*}\right)}}=e(g, g)^{s}
$$

The message then can be obtained as : $\mathcal{M}=C_{0} / e(g, g)^{s}$.

To see the soundness of the Decryption algorithm observe the following:

$$
\begin{aligned}
\mathscr{A} & =\prod_{x}\left(\frac{C_{1, x} \cdot e\left(H(G I D), C_{3, x}\right)}{e\left(K_{\rho(x), G I D}, C_{2, x}\right)}\right)^{c_{x}}=\prod_{x}\left(e(g, g)^{\lambda_{x}+\omega_{x} \log _{g} H(G I D)}\right)^{c_{x}} \\
& =e(g, g)^{\sum_{x} \lambda_{x} c_{x}} \cdot e(H(G I D), g)^{\sum_{x} \omega_{x} c_{x}}=e(g, g)^{s+s^{*} \log _{g} H(G I D)} \\
\mathscr{B} & =\prod_{k=1}^{r}\left(e\left(K_{G I D}^{*}, C_{2, k}^{*}\right) e\left(C_{1, k}^{*}, H(G I D)\right)\right)^{-1 /\left(G I D-G I D_{k}^{*}\right)} \\
& =\prod_{k=1}^{r}\left(e(g, g)^{\left(G I D-G I D_{k}^{*}\right) s_{k} \log _{g} H(G I D)}\right)^{-1 /\left(G I D-G I D_{k}^{*}\right)} \\
& =e(g, g)^{-\sum_{k=1}^{r} s_{k} \log _{g} H(G I D)}=e(g, g)^{-s^{*} \log _{g} H(G I D)}
\end{aligned}
$$

Remarks. (i) We note that an almost equivalent result can be achieved, with some different modifications on the decentralized scheme (splitting $C_{1, x}$ into two parts, using $e(g, g)^{\beta s}$ for encryption, where $\beta$ is the secret of the CA, and publishing $g^{s}$ ) and fitting it to the method of [LZW ${ }^{+} 13$ ]. However in this way additional modifications are still needed to prevent the CA from being able to decrypt any ciphertext by computing $e\left(g^{\beta}, g^{s}\right)$.

(ii) Supposing that we have a honest but curious CSP, which does not collude with the users, it is also possible to achieve indirect revocation (similarly to [LLLS10|SSW12]), with simple modifications on our scheme. With other words, the CSP could fully supervise user revocation based on the revocation requests from parties, authorised for this. We only need to modify the Encrypt algorithm to compute $C, C_{0}, C_{1, x}, C_{2, x}$ as originally and $C_{3, x}^{\prime}=g^{y_{\rho(x)} r_{x}} \quad \forall x=1, \ldots, n$. These values would form $C T^{\prime}$ that is sent to the CSP, where the collusion resistant $C T$ with the revocation information is computed and published. $C T$ has the same form as earlier, the only difference is that the blinding vector $w$ is chosen by the CSP, so $\omega_{x}, C_{1, k}^{*}, C_{2, k}^{*}$ (as previously) and $C_{3, x}=C_{3, x}^{\prime} \cdot g^{\omega_{x}}$ are computed also by the CSP. The main advantage of this approach is that immediate and efficient (partial) re-encryption can be achieved as only $w, s_{k}, \omega_{x}, C_{1, k}^{*}, C_{2, k}^{*}$ and $C_{3, x}$ need to be recomputed after a revocation event.

(iii) Alternatively, it is also possible to give revocation right directly to the encryptor by simply publishing a user list instead of $R L$. In this case $R L$ would be defined by the user, separately for each ciphertext, and attached to $C T$. 


\subsection{Efficiency}

Traditional, attribute-based user revocation (e.g. [WLWG11|RNS11|YJRZ13]) affects attributes, thus the revocation of a user may cause the update of all the users' attribute secret keys who had common attribute with the revoked user (a general attribute can affect big proportion of the users) and the re-encryption of all ciphertext the access structure of which contain any of the revoked user's attributes (most of these could not be decrypted by the revoked user).

In our scheme, a revocation event does not have any effect on the attributes as it is based on identity. Although it is a trade-off and in the other hand there is some computational overhead on the encryption and decryption algorithms. In this way the necessary extra computation of authorities is reduced and distributed between the largest set of parties, the users, preventing a possible performance bottleneck of the system. At the same time the extra communication is also reduced to the publication of the revoked user list. Our revocation scheme has the following costs.

The ciphertext has $2 r$ additional elements, if the number of revoked users is $r$. For the computation of these values $3 r$ exponentiations and $r$ multiplications are needed in $\mathbb{G}_{0}$. Alternatively, the revoked user list may contain $g^{a} g^{G I D_{i}^{*}}$ instead of the global identifiers. In this case the encryptor need to do only $2 r$ additional exponentiations in $\mathbb{G}_{0}$, compared with the scheme of [LW11, to compute the ciphertext. The overhead of the decryption algorithm is $2 r$ pairing operations, $r$ multiplications and exponentiations in group $\mathbb{G}_{1}$.

Note that, as in all model that uses LSSS to express the access structure, the access matrix and the mapping $\rho$ must be part of the ciphertext, increasing its length. However, it is possible to reduce this length by attaching only a formatted Boolean formula instead and compute the necessary components of LSSS more efficiently, using the algorithm of Liu and Cao in LC10.

\subsection{Security}

Before giving the formal proof, we point out that from the point of view of a user, whose attributes have never satisfied the access structure defined in the ciphertext, our construction is at least as secure as the one by [LW11, because the computation of $\mathscr{A}$ is equivalent to the decryption computation given there. However in our case, it is not enough to obtain the message. Changing the first entry of the blinding vector $w$ from zero to a random number (as we did), causes that the blinding will not cancel out from $\mathscr{A}$, but we need to compute $\mathscr{B}$ which can divide it out. $\mathscr{B}$ can be computed with any $G I D$ different from any $G I D_{k}^{*}$ of the revocation list and we ensure that the decryptor must use the same $G I D$ both in $\mathscr{A}$ and $\mathscr{B}$ by using $H(G I D)$ in both the identity and attribute secret keys.

We are going to prove the security of our construction in the generic bilinear group model previously used in [BBG05|BSW07|LW11], modelling $H$ as a random oracle. Security in this model assures us that an adversary cannot break our scheme with only black-box access to the group operations and $H$. At an intuitive level, this means that if there are any vulnerabilities in our scheme, then these vulnerabilities must exploit specific mathematical properties of elliptic curve groups or cryptographic hash functions used when instantiating our construction.

We describe the generic bilinear model as in BBG05. We let $\psi_{0}$ and $\psi_{1}$ be two random encodings of the additive group $\mathbb{Z}_{p}$. More specifically, each of $\psi_{0}, \psi_{1}$ is an injective map from $\mathbb{Z}_{p}$ to $\{0,1\}^{m}$, for $m>3 \log (p)$. We define the groups $\mathbb{G}_{0}=\left\{\psi_{0}(x): x \in \mathbb{Z}_{p}\right\}$ and $\mathbb{G}_{1}=\left\{\psi_{1}(x): x \in \mathbb{Z}_{p}\right\}$. We assume we have access to oracles which compute the induced group operations in $\mathbb{G}_{0}$ and $\mathbb{G}_{1}$ and an oracle which computes a non-degenerate bilinear map $e: \mathbb{G}_{0} \times \mathbb{G}_{0} \rightarrow \mathbb{G}_{1}$. We refer to $\mathbb{G}_{0}$ as a generic bilinear group. To simplify our notations let $g$ denote $\psi_{0}(1), g^{x}$ denote $\psi_{0}(x), e(g, g)$ denote $\psi_{1}(1)$, and $e(g, g)^{y}$ denote $\psi_{1}(y)$.

Theorem 1. For any adversary $\mathcal{A}$, let $q$ be a bound on the total number of group elements it receives from queries it makes to the group oracles and from its interaction with the security game, described in 2.5. The above described construction is secure according to Definition 3 in the generic bilinear group and random oracle models. The advantage of $\mathcal{A}$ is $\mathcal{O}\left(q^{2} / p\right)$.

Proof. In our security game, $\mathcal{A}$ must distinguish $C_{0}=M_{0} e(g, g)^{s}$ from $C_{0}=M_{1} e(g, g)^{s}$. We can alternatively consider a modified game, where the attacker must distinguish between $C_{0}=e(g, g)^{s}$ 
or $C_{0}=e(g, g)^{T}$, for $T$ chosen uniformly randomly from $\mathbb{Z}_{p}$. This is the same modification employed in BSW07/LW11, and it is justified by a simple hybrid argument.

We now simulate the modified security game in the generic bilinear group model where $C_{0}$ is set to be $e(g, g)^{T}$. We let $S$ denote the set of all authorities, $U$ the universe of attributes and $R L$ the Revocation List. The simulator runs the global setup algorithm, and gives $g$ to the attacker. $\mathcal{A}$ chooses a set $S^{\prime} \subset S$ of corrupted authorities, and reveals these to the simulator. The simulator randomly chooses values $a, b \in \mathbb{Z}_{p}$ for the identity key generation and $\alpha_{i}, y_{i} \in \mathbb{Z}_{p}$ for the attributes $i \in U$ controlled by uncorrupted authorities, and it queries the group oracles for $g^{a}, g^{1 / b}$ and for each $g^{y_{i}}, e(g, g)^{\alpha_{i}}$ and gives these to the attacker. When the attacker requests $H\left(G I D_{k}\right)$ for some $G I D_{k}$ for the first time, the simulator chooses a random value $h_{G I D_{k}} \in \mathbb{Z}_{p}$, queries the group oracle for $g^{h_{G I D_{k}}}$, and gives this value to the attacker as $H\left(G I D_{k}\right)$. It stores this value so that it can reply consistently to any subsequent requests for $H\left(G I D_{k}\right)$.

Upon a request for $K_{G I D_{k}}^{*}$ for some $G I D_{k}$ the simulator uses the group oracle to compute $g^{\left(G I D_{k}^{*}+a\right) b h_{G I D_{k}}}$ and supplies this value to the attacker. A request for a key $K_{i, G I D_{k}}$ for some attribute $i$ and identity $G I D_{k}$ handled similarly, $g^{\alpha_{i}} H\left(G I D_{k}\right)^{y_{i}}$ is computed using the group oracle and sent to the attacker. In both cases, if $H\left(G I D_{k}\right)$ has not been requested before, it is determined as above.

At some point, the attacker specifies an access matrix $(A, \rho)$ for the challenge ciphertext and additionally supplies the simulator with the $g^{y_{i}}, e(g, g)^{\alpha_{i}}$ values for any attributes $i$ controlled by corrupt authorities that appear in the image of $\rho$ on the rows of $A$. The simulator then checks that these are valid group elements by querying the group oracles.

The simulator must now produce the challenge ciphertext. To do so, it chooses random values $s, v_{2}, \ldots, v_{\ell}, s^{*}, w_{2}, \ldots, w_{\ell} \in \mathbb{Z}_{p}$, sets the sharing vector $v=\left(s, v_{2}, \ldots, v_{\ell}\right)$ and computes the shares $\lambda_{x}=A_{x} \cdot v$. Similarly it set the blinding vector $w=\left(s^{*}, w_{2}, \ldots, w_{\ell}\right)$ and computes $\omega_{x}=A_{x} \cdot w$. Random values $r_{x} \in \mathbb{Z}_{p}$ are chosen for each row $A_{x}$ of $A$, and a random value $T \in \mathbb{Z}_{p}$. The values of $s_{1}, \ldots, s_{r-1} \in \mathbb{Z}_{p}$ are also chosen randomly, while $s_{r}=s^{*}-\sum_{i=1}^{r-1} s_{i}$ (where $r=|R L|$ ). Using the group oracles, the simulator can now compute:

$$
\begin{array}{cc}
C_{0}=e(g, g)^{T} \\
\forall x=1, \ldots, n: & C_{1, x}=e(g, g)^{\lambda_{x}} e(g, g)^{\alpha_{\rho(x)} r_{x}}, C_{2, x}=g^{r_{x}}, C_{3, x}=g^{y_{\rho(x)} r_{x}} g^{\omega_{x}} \\
\forall k=1, \ldots, r: & C_{1, k}^{*}=\left(g^{a} g^{G I D_{k}^{*}}\right)^{-s_{k}}, C_{2, k}^{*}=g^{s_{k} / b}
\end{array}
$$

and the challenge ciphertext is given to the attacker.

We will argue that will all but negligible probability, the attacker's view in the simulation is identically distributed to what it's view would have been if $C_{0}$ had been set to $e(g, g)^{s}$ instead of $e(g, g)^{T}$. This shows that the attacker cannot attain a non-negligible advantage in the modified security game, and hence in the real one.

We condition on the event that each of the attacker's queries to the group oracles have input values that were given to the attacker during the simulation or were received from the oracles in response to previous queries. Since each $\psi_{0}, \psi_{1}$ is a random injective map from $\mathbb{Z}_{p}$ into a set of $>p^{3}$ elements, the probability of the attacker being able to guess an element in the image of $\psi_{0}, \psi_{1}$ which it has not previously obtained is negligible.

Under this condition, we can think of each of the attacker's queries as a multi-variate expressions in the variables $T, y_{i}, \alpha_{i}, \lambda_{x}, r_{x}, \omega_{x}, h_{G I D_{k}}, a, b, s_{k}$, where $i$ ranges over the attributes controlled by uncorrupted authorities, $x$ ranges over the rows of the challenge access matrix, $k$ ranges over the revoked identities. (We can also think of $\lambda, \omega_{x}$ as linear combinations of the variables $s, v_{2}, \ldots, v_{\ell}$ and $s^{*}, w_{2}, \ldots, w_{\ell}$.)

We now further condition on the event that for each pair of queries $\mathcal{A}$ makes corresponding to different polynomials, it receives different answers. In other words, we are conditioning on the event that our random assignment of values to the previous variables does not happen to be a zero of the difference of two query polynomials. Since our polynomials have degree at most 8 (see the possible polynomials later), using the Schwartz-Zippel lemma we have that the probability of a collusion is $\mathcal{O}(1 / p)$ and a union bound shows that the probability of that any such collusion happens during the simulation is $\mathcal{O}\left(q^{2} / p\right)$. Now suppose that it does not happen.

Since $T$ only appears as $e(g, g)^{T}$, the only queries the attacker can make involving $T$ are of the form $c T+$ other terms, where $c$ is a constant. The attacker's view can only differ when $T=s$ if the attacker can make two queries $f$ and $f^{\prime}$ into $\mathbb{G}_{1}$ where these are unequal as polynomials but 
Table 2. Possible relevant query terms

\begin{tabular}{|c|c|}
\hline$s_{k} a+G I D_{k}^{*} s_{k}$ & $s_{k} / b$ \\
\hline$s_{k} s_{l} a^{2}+G I D_{k}^{*} G I D_{l}^{*} s_{k} s_{l}+\left(G I D_{k}^{*}+G I D_{l}^{*}\right) s_{k} s_{l} a$ & $s_{k} s_{l} / b^{2}$ \\
\hline$s_{k} a^{2}+G I D_{k}^{*} s_{k} a$ & $s_{k} a / b$ \\
\hline$s_{k} a / b+G I D_{k}^{*} s_{k} / b$ & $s_{k} / b^{2}$ \\
\hline$s_{k} b h_{G I D_{l}}\left(a^{2}+\left(G I D_{k}^{*}+G I D_{l}^{*}\right) a+G I D_{k}^{*} G I D_{l}^{*}\right)$ & $s_{k} a h_{G I D_{l}}+G I D_{l}^{*} s_{k} h_{G I D_{l}}$ \\
\hline$s_{k} a h_{G I D_{l}}+G I D_{k}^{*} s_{k} h_{G I D_{l}}$ & $s_{k} h_{G I D_{l}} / b$ \\
\hline$s_{k} s_{l} a / b+G I D_{k}^{*} s_{k} s_{l} / b$ \\
\hline \multicolumn{2}{|c|}{} \\
\hline \multicolumn{2}{|c|}{}
\end{tabular}

become the same when we substitute $s$ for $T$. This implies $f-f^{\prime}=c s-c T$ for some constant $c$. We may conclude that the attacker can then make the query $c s$.

We will now show that the attacker cannot make a query of the form $c s$, and therefore arrive at a contradiction. By examining the values given to the attacker during the simulation, [LW11] showed that without a satisfying set of attributes an attacker cannot make a query of the form $c(s+$ $\left.0 \cdot h_{G I D_{k}}\right)$ thus has only a negligible advantage in distinguishing an encoded message from a random group element (in their original scheme). This result implies that in our modified construction, the attacker cannot make a query of the form $c\left(s+s^{*} h_{G I D_{k}}\right)$ without a satisfying set of attributes (as the first element of the blinding vector $w$ is changed to $s^{*}$ from zero) which also shows - following the their reasoning - that cs cannot be formed either. In the other hand, in our case the possession of the necessary attributes are not enough to make a $c s$ query, but $-c\left(s^{*} h_{G I D_{k}}\right)$ is also indispensable for this. From now on we assume that $G I D_{k} \in R L$ thus the challenge access structure is satisfied (and simulate that all revoked users are colluding), as the case when $G I D_{k} \in U L \backslash R L$ is equivalent to the original scheme of [LW11. We will prove that $\mathcal{A}$ cannot be successful by showing it cannot make a query of the form $-c\left(s^{*} h_{G I D_{k}}\right)$ and so not $c s$.

We see that the attacker can form queries which are linear combinations of

$$
\begin{gathered}
1, h_{G I D_{k}}, y_{i}, \alpha_{i}+h_{G I D_{k}} y_{i}, \lambda_{x}+\alpha_{\rho(x)} r_{x}, r_{x}, y_{\rho(x)} r_{x}+\omega_{x} \\
a, 1 / b, b h_{G I D_{k}}\left(G I D_{k}^{*}+a\right), s_{k}\left(a+G I D_{k}^{*}\right), s_{k} / b
\end{gathered}
$$

the product of any two of these and $\alpha_{i}, T$. (Note that $G I D_{k}^{*}$ for all $k=1, \ldots, r$ and $\alpha_{i}, y_{i}$ for attributes $i$ controlled by corrupted authorities are constants, known by the attacker.) In these queries $s^{*}$ can appear in two different forms: as $\omega_{x}$ and $s_{k}$.

In order to gain $s^{*} h_{G I D_{k}}$ by utilizing $\omega_{x}, \mathcal{A}$ must use the product $h_{G I D_{k}} y_{\rho(x)} r_{x}+h_{G I D_{k}} \omega_{x}$ for all rows of $A$, as these are the only terms which contain $h_{G I D_{k}} \omega_{x}$ the proper linear combination of which leads to $s^{*} h_{G I D_{k}}$. To cancel out $h_{G I D_{k}} y_{\rho(x)} r_{x}$ the attacker should form this product, which is possible only if $y_{\rho(x)}$ or $r_{x}$ are known constants as otherwise the needed elements appear alone in the above list and besides those, $\mathcal{A}$ can only form the product of any two but not three. However if $y_{\rho(x)}$ or $r_{x}$ are constants for all $x$, that contradicts with the rules of the security game as only corrupted attributes would satisfy the access structure.

On $s_{k}$, we can make the following observations. (1) In each term, $s_{k}$ appears as multiplier either in all monads or in none of them. (2) To form $c \cdot s_{k} h_{G I D_{l}}$ (for a chosen $l$ and all $k$ ) as linear combination of different terms, these must contain $s_{k}$ as multiplier, so terms without $s_{k}$ are useless (see Table 2 for the possible query terms). (3) In the linear combination there must be a term which contains $s_{k} h_{G I D_{l}}$ maybe multiplied with some constant.

As it can be seen in Table 2 there are two terms which contain the necessary monad:

$$
s_{k} a h_{G I D_{l}}+G I D_{k}^{*} s_{k} h_{G I D_{l}} \text { and } s_{k} a h_{G I D_{l}}+G I D_{l}^{*} s_{k} h_{G I D_{l}},
$$

multiplied each by $c /\left(G I D_{k}^{*}-G I D_{l}^{*}\right)$ it is possible to gain $c \cdot s_{k} h_{G I D_{l}}$, if $k \neq l$. However in case of $k=l$ the two terms are equal, and $s_{k} a h_{G I D_{l}}$ cannot be cancelled out, as no other terms contain this product. We conclude that it is possible to gain $s_{k} h_{G I D_{l}}$ for all $k$ (thus $-c \sum_{k=1}^{r} s_{k} h_{G I D_{l}}$ ) if and only if there exists $l$ which is not from the same set, as $k$. Here we arrive at a contradiction as both $k, l \in 1, \ldots, r$, otherwise the attacker would have used some $G I D_{l} \notin R L$.

Hence, we have shown that the attacker cannot construct a query of the form $c s$ for a constant c. Therefore, under conditions that hold with all but $\mathcal{O}\left(q^{2} / p\right)$ probability, the attacker cannot distinguish between the cases when $T$ is random or $T=s$ thus the advantage of $\mathcal{A}$ in the security game is at most $\mathcal{O}\left(q^{2} / p\right)$. 


\section{Future Work}

We proposed a scheme for efficient identity-based user revocation in multi-authority CP-ABE. In the future, our work can be continued in several directions.

First and foremost, extensive comparisons are needed between the different revocation schemes proposed for attribute-based encryption to understand better their performance between different circumstances.

Securely forwarding the revocation related computations to the CSP (or even to the user), as we mentioned in a remark, could allow immediate banning of a user, disallowing the decryption of all previously (and later) encrypted ciphertexts. Steps in this direction, without assuming trusted CSP, would be useful.

The method of identity-based user revocation can be the foundation of a future method that allows non monotonic access structures in multi-authority setting. However our scheme cannot be applied directly for this purpose, it may be used to develop ideas in this field.

The security of our construction is proved in the generic bilinear group model, although we believe it would be possible to achieve full security by adapting the dual system encryption methodology, which was also used by Lewko and Waters [LW11] in their composite order group construction. This type of work would be interesting even if it resulted in a moderate loss of efficiency from our existing system.

Acknowledgements. This work was started as a master thesis at Eötös Loránd University, in the Security\&Privacy program of EIT ICT Labs Masterschool. I would like to express my gratitude to my supervisor, Levente Buttyán from CrySyS Lab for all his help and valuable advice. I am also grateful to Viktória Villányi and Péter Ligeti for the useful discussions and to the anonymous reviewer of SOFSEM'15 for the valuable remarks.

\section{References}

BBG05. Dan Boneh, Xavier Boyen, and Eu-Jin Goh. Hierarchical identity based encryption with constant size ciphertext. In Advances in Cryptology-EUROCRYPT 2005, pages 440-456. Springer, 2005. 9

Bei96. Amos Beimel. Secure schemes for secret sharing and key distribution. PhD thesis, Israel Institute of Technology, Technion, Haifa, Israel, 1996. 3, 4

BSW07. John Bethencourt, Amit Sahai, and Brent Waters. Ciphertext-policy attribute-based encryption. In IEEE Symposium on Security and Privacy, pages 321-334, 2007. 2, 9,10

Cha07. Melissa Chase. Multi-authority Attribute Based Encryption. In Theory of Cryptography, volume 4392 of $L N C S$, pages 515-534. Springer Berlin Heidelberg, 2007. 2

CL14. Zhengjun Cao and Lihua Liu. Analysis of Lewko-Sahai-Waters Revocation System. Cryptology ePrint Archive, Report 2014/937, 2014. http://eprint.iacr.org/. 6

LC10. Zhen Liu and Zhenfu Cao. On Efficiently Transferring the Linear Secret-Sharing Scheme Matrix in Ciphertext-Policy Attribute-Based Encryption. IACR Cryptology ePrint Archive, 2010:374, 2010. 9

LLLS10. Xiaohui Liang, Rongxing Lu, Xiaodong Lin, and Xuemin Sherman Shen. Ciphertext policy attribute based encryption with efficient revocation. TechnicalReport, University of Waterloo, 2010. 2,8

LSW10. Allison Lewko, Amit Sahai, and Brent Waters. Revocation systems with very small private keys. In IEEE Symposium on Security and Privacy, pages 273-285, 2010. 2, 6

LW11. Allison Lewko and Brent Waters. Decentralizing attribute-based encryption. In Advances in Cryptology-EUROCRYPT 2011, pages 568-588. Springer, 2011. 1, 2, 4, 5, 6, 7, 9, 10, 11, 12

LXZ13. Qinyi Li, Hu Xiong, and Fengli Zhang. Broadcast revocation scheme in composite-order bilinear group and its application to attribute-based encryption. International Journal of Security and Networks, 8(1):1-12, 2013. 2

$\mathrm{LZW}^{+}$13. Yang Li, Jianming Zhu, Xiuli Wang, Yanmei Chai, and Shuai Shao. Optimized CiphertextPolicy Attribute-Based Encryption with Efficient Revocation. International Journal of Security E Its Applications, 7(6), 2013. 2, 8

QD11. Jun-lei Qian and Xiao-lei Dong. Fully secure revocable attribute-based encryption. Journal of Shanghai Jiaotong University (Science), 16:490-496, 2011. 2 
RNS11. Sushmita Ruj, Amiya Nayak, and Ivan Stojmenovic. Dacc: Distributed access control in clouds. In IEEE 10th International Conference on Trust, Security and Privacy in Computing and Communications, pages 91-98, 2011. 2, 9

SSW12. Amit Sahai, Hakan Seyalioglu, and Brent Waters. Dynamic credentials and ciphertext delegation for attribute-based encryption. In Advances in Cryptology-CRYPTO 2012, pages 199-217. Springer, 2012. 2, 8

SW05. Amit Sahai and Brent Waters. Fuzzy identity-based encryption. In Advances in CryptologyEUROCRYPT 2005, pages 457-473. Springer, 2005. 1, 2

Wat11. Brent Waters. Ciphertext-policy attribute-based encryption: An expressive, efficient, and provably secure realization. In Public Key Cryptography-PKC 2011, pages 53-70. Springer, 2011. 2

WLWG11. Guojun Wang, Qin Liu, Jie Wu, and Minyi Guo. Hierarchical attribute-based encryption and scalable user revocation for sharing data in cloud servers. Computers $\&$ Security, 30(5):320-331, 2011. 2, 9

YJRZ13. Kan Yang, Xiaohua Jia, Kui Ren, and Bo Zhang. DAC-MACS: Effective data access control for multi-authority cloud storage systems. In INFOCOM, 2013 Proceedings IEEE, pages 28952903, 2013. 29 